\title{
Bipolar Models for a More Realistic Representation and Processing of Human Judgments, Intentions and Preferences: A Role of Fuzzy Logic
}

\author{
Janusz Kacprzyk $^{(\otimes)}$ \\ Systems Research Institute, Polish Academy of Sciences, ul. Newelska 6, \\ 01-447 Warsaw, Poland \\ kacprzyk@ibspan.waw.pl
}

We deal with a grand challenge facing IT/ICT the essence of which is to make computers to be "cognitive partners" for humans. Our focus is decision making, a "metaproblem" in virtually all human activities. Decisions are made by the humans, and for the humans, even if mimicked by/in inanimate systems, and this should be accounted for in tools and techniques to be employed. In most real life situation decision making should be augmented by decision aiding or decision support systems.

Decision making, in the formal setting, boils down to finding a best decision, usually using some choice or optimization tools. Multiple criteria, decision makers, dynamics, etc. can be added. Imperfect information is crucial, and this was traditionally equated with probabilistic uncertainty. Extensions to deal with imprecise information are here relevant and will be dealt with here. Notably with respect to judgments, attitudes and intentions of the (human) agents. More specifically, tools to deal with uncertain and imprecise (fuzzy) preferences and utilities, bipolar assessments by "pro" and "con" arguments and conditions, tools for expressing coordination, cooperation and collaboration, as well as a full range of general attitudes: from greed to fairness, maybe even to the altruism, would be important. A crucial issue of context is also important.

We assume first that we operate in the context of show how to formalize and solve problems in which there is a bipolarity in the decision maker's judgments, intentions and preferences which is related to the inclusion in real human judgments what is good and bad, i.e. pros and cons, what is necessary and optional, what should result in rejection and acceptance, etc. The inclusion of these features leads to new models that can be meant as a broadly perceived bipolar decision making models. Their essence will be shown with examples mostly in multicriteria decision making and database querying, Hierarchical representations of more abstract and complex pro and con arguments will be dealt with. Some ways of accounting for context will be shown. As an effective and efficient tool fuzzy logic will be advocated.

Fellow of IEEE, IET, IFSA, EurAI/ECCAI, SMIA, Full Member, Polish Academy of Sciences, Member, Academia Europaea, Member, European Academy of Sciences and Arts, Foreign Member, Bulgarian Academy of Sciences, Foreign Member, Spanish Royal Academy of Economic and Financial Sciences (RACEF).

(C) Springer Nature Switzerland AG 2020

C. Kahraman et al. (Eds.): INFUS 2019, AISC 1029, p. 3, 2020.

https://doi.org/10.1007/978-3-030-23756-1_1 